\title{
Lacunar Infarction Associated with Anabolic Steroids and Polycythemia: A Case Report
}

\author{
George W.J. Harston ${ }^{a}$ Farzana Batt ${ }^{c}$ Lampson Fan ${ }^{c}$ Thomas W. Okell ${ }^{b}$ \\ Fintan Sheerin $^{d}$ Timothy Littlewood $^{\mathrm{e}}$ James Kennedy $^{\mathrm{a}}$ \\ ${ }^{a}$ Radcliffe Department of Medicine, and ${ }^{b}$ FMRIB, Nuffield Department of Clinical \\ Neurosciences, University of Oxford, 'Acute Stroke Service, and Departments of \\ ${ }^{\mathrm{d}}$ Neuroradiology and ${ }^{\mathrm{e}} \mathrm{Clinical}$ Haematology, Oxford University Hospitals NHS Trust, \\ Oxford, UK
}

\section{Key Words}

Cerebral infarction · Steroids · Polycythemia

\begin{abstract}
Lacunar infarction is traditionally ascribed to lipohyalinosis or microatheroma. We report the case of 40-year-old man, without traditional risk factors for ischemic stroke, who presented to the Emergency Department with recurrent episodes of transient right-sided weakness and paresthesia. Lacunar infarction was confirmed on diffusion-weighted MRI and blood tests showed a marked polycythemia. Quantitative magnetic resonance perfusion imaging demonstrated dramatically abnormal perfusion throughout both cerebral hemispheres, and transcranial Doppler revealed reduced cerebral artery velocities, both consistent with the proposed mechanism of hyperviscosity. His symptoms settled with treatment of the polycythemia and workup did not find another cause of ischemic stroke. We propose that hyperviscosity secondary to steroid-induced polycythemia caused ischemia in this case and not lipohyalinosis or microatheroma, to which lacunar disease is commonly attributed.
\end{abstract}

(c) 2014 S. Karger AG, Basel

\section{Introduction}

Focal cerebral manifestations of polycythemia have been previously described in the context of polycythemia vera [1] and other causes of secondary polycythemia [2]. We report 
Harston et al.: Lacunar Infarction Associated with Anabolic Steroids and Polycythemia: A Case Report

a case where the use of anabolic androgenic steroids (AAS) for bodybuilding was associated with polycythemia and lacunar infarction.

\section{Case Presentation}

A 40-year-old bodybuilder presented to the Emergency Department following 2 episodes of right-sided weakness and paresthesia on the same day, each lasting $10 \mathrm{~min}$. The patient admitted regular use of AAS for bodybuilding (2a-17a-dimethyl-17b-hydroxy-5aandrostan-3-one, stanozolol, testosterone and trenbolone). Past medical history was significant for steroid-induced cholestasis 6 years previously. Of note, he was not hypertensive at the time of that presentation. Outside of a 5 pack-year history of tobacco smoking, there was no history of traditional cardiovascular risk factors. There was no history of erythropoietin use. He denied other drug use, including cocaine.

On arrival, his blood pressure was $210 / 100 \mathrm{~mm} \mathrm{Hg}$ and oxygen saturation was $94 \%$ on air. His symptoms had resolved, and mentation and neurological examination were normal. There was no clinical evidence of hypertensive end-organ damage. Baseline laboratory investigations showed an elevated hematocrit of $56.9 \%$ and hemoglobin concentration of $20.6 \mathrm{~g} / \mathrm{dl}$ (normal range: $13-17 \mathrm{~g} / \mathrm{dl}$ ). Both had been normal 6 years previously. Other than a mildly elevated alanine transaminase, the remainder of the initial laboratory investigations was normal, including ECG, white cell and platelet counts, kidney function, and toxicology screen including cocaine metabolites.

Brain CT scan and intracranial CT angiogram were normal. Brain MRI showed restricted diffusion in the posterior limb of the left internal capsule. Quantitative arterial-spin labelling perfusion MRI demonstrated an abnormal pattern of perfusion signal with reduced cerebral blood flow throughout the cerebral hemispheres bilaterally (fig. 1). Transcranial Doppler (TCD) examination revealed global reductions in flow velocities $(75 \%$ of mean velocity at follow-up post-venesections).

Over the following week, the patient suffered 2 further transient ischemic events with similar clinical features. After each episode he was venesected, and $450 \mathrm{ml}$ of blood was removed. After venesection, his symptoms settled and the hematocrit returned to the normal range.

Brain MRI at 1 month demonstrated mature infarction in the posterior limb of the left internal capsule. Carotid Doppler ultrasound was normal. Workup for polycythemia showed a normal erythropoietin concentration (with concurrent normal hemoglobin concentration), normal arterial blood gases, normal vasculitis and thrombophilia screens, and no JAK2 mutation. Abdominal ultrasound demonstrated normal kidneys, liver and spleen. Three months following cessation of AAS, the patient had no further symptoms and his hematocrit was normal without further venesection. Blood pressure was $120 / 80 \mathrm{~mm} \mathrm{Hg}$ without antihypertensive medication.

\section{Discussion}

A case of sensorimotor transient ischemic attack is described in keeping with the demonstrated infarction of the posterior limb of the internal capsule confirmed on both acute diffusion-weighted imaging and follow-up FLAIR imaging. The patient had no evidence of macroatheroma on imaging and, other than a 5 pack-year smoking history, had no traditional risk factors for ischemic stroke. AAS are known to induce polycythemia, although 
the precise mechanism is not clear, but variably ascribed to direct effects on the hematopoietic stem cells and to increased erythropoietin production. We propose that increased blood viscosity, secondary to AAS-induced polycythemia in this case, directly contributed to cerebral infarction, evidenced by the abnormal perfusion imaging, reduced middle cerebral artery velocities and lack of traditional risk factors for lacunar infarction.

Interestingly, in this case it is recurrent focal episodes that manifested symptomatically rather than diffuse impairment of cerebral function. Focal infarction may be the end result of the 'viscous, vicious circle' of a low flow state and red cell aggregation [3]. Apparent blood viscosity is a function of the shear rate of blood. In a low flow state (demonstrated in this case by globally reduced flow velocities on TCD), a low shear rate leads to red cell aggregation, including rouleaux formation, with increased apparent viscosity. This results in reduced cerebral perfusion (see fig. 1 demonstrating global reductions in cerebral blood flow) [4, 5]. An increased hematocrit magnifies the effects of low sheer rate resulting in a logarithmic increase in apparent blood viscosity. This triggers a cycle of further reductions in flow and increased red cell aggregation with eventual stasis and thrombus formation.

The limitation of this case is that the spatial resolution of 3T MRI is insufficient to rule out the traditional causes of lacunar infarction, namely lipohyalinosis or microatheroma. However, the correlation of symptoms with hematocrit, reduced arterial-spin labelling perfusion signal and reduced large artery flow velocities on TCD support the hypothesis that hyperviscosity from AAS-induced polycythemia contributed to the mechanism of acute lacunar infarction in this patient.

\section{Acknowledgements}

This research was supported by the National Institute for Health Research (NIHR) Oxford Biomedical Research Centre Programme, the Dunhill Medical Trust (grant No. OSRP1/1006) and the Centre of Excellence for Personalized Healthcare funded by the Wellcome Trust and EPSRC under grant No. WT 088877/Z/09/Z. We wish to acknowledge the facilities provided by the Oxford Acute Vascular Imaging Centre.

\section{Disclosure Statement}

Dr. T.W. Okell received royalties from Siemens Healthcare for the licensing of two pending US patents.

\section{References}

1 Barabas AP, Offen DN, Meinhard EA: The arterial complications of polycythaemia vera. Br J Surg 1973;60:183-187.

2 Thakur KT, Westover MB: Cerebral infarction due to smoker's polycythemia. BMJ Case Rep 2011;2011:bcr0820114714.

-3 Thomas DJ: Whole blood viscosity and cerebral blood flow. Stroke 1982;13:285-287.

4 Koenig W, Ernst E: The possible role of hemorheology in atherothrombogenesis. Atherosclerosis 1992;94:93-107.

5 Somer T, Meiselman HJ: Disorders of blood viscosity. Ann Med 1993;25:31-39.

6 Bristow MS, Simon JE, Brown RA, Eliasziw M, Hill MD, Coutts SB, Frayne R, Demchuk AM, Mitchell JR: MR perfusion and diffusion in acute ischemic stroke: human gray and white matter have different thresholds for infarction. J Cereb Blood Flow Metab 2005;25:1280-1287. 
Harston et al.: Lacunar Infarction Associated with Anabolic Steroids and Polycythemia: A Case Report
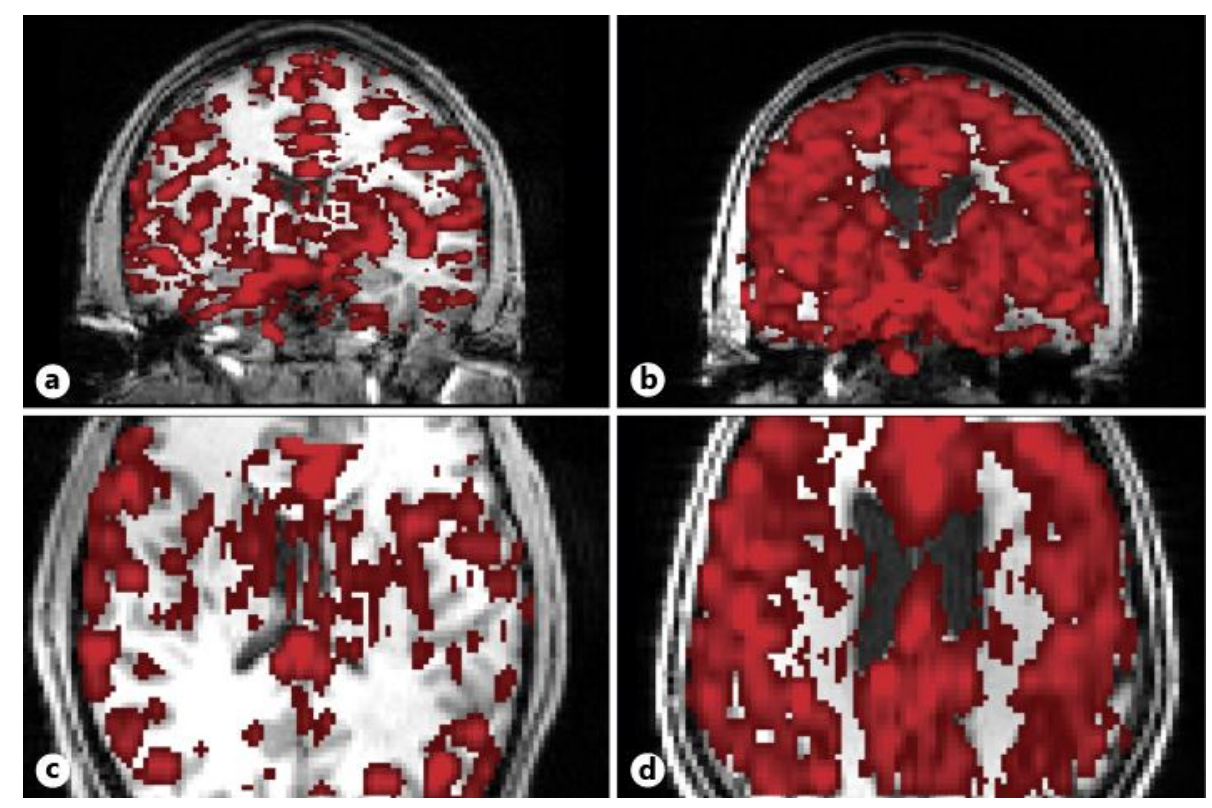

Fig. 1. Coronal and axial sections of arterial-spin labelling perfusion imaging overlaid on a co-registered T1-weighted image of the case patient $(\mathbf{a}, \mathbf{b})$ compared to a control patient $(\mathbf{c}, \mathbf{d})$ also with lacunar stroke within $6 \mathrm{~h}$ of symptom onset, but with a normal hematocrit. Perfusion imaging is thresholded at 12.3 $\mathrm{ml} / 100 \mathrm{~g} / \mathrm{min}[6]$. 